04

\title{
Анализ потерь быстрых ионов, вызванных распространением тороидальных альфвеновских мод в плазме сферического токамака Глобус-М2
}

\author{
() И.М. Балаченков, Н.Н. Бахарев, В.И. Варфоломеев, В.К. Гусев, М.В. Ильясова, Г.С. Курскиев, \\ В.Б. Минаев, М.И. Патров, Ю.В. Петров, Н.В. Сахаров, О.М. Скрекель, А.Ю. Тельнова, \\ Е.М. Хилькевич, А.Е. Шевелев, П.Б. Щеголев
}

Физико-технический институт им. А.Ф. Иофрфе РАН, 194021 Санкт-Петербург, Россия e-mail: balachenkov@mail.ioffe.ru

Поступило в Редакцию 2 августа 2021 г. В окончательной редакции 9 октября 2021 г. Принято к публикации 10 октября 2021 г.

\begin{abstract}
С увеличением магнитного поля до $0.8 \mathrm{~T}$ и тока по плазме до $0.4 \mathrm{MA}$ в токамаке Глобус-М2 уровень потерь быстрых ионов в разрядах с тороидальными альфвеновскими модами (ТАЕ) снизился по сравнению с установкой Глобус-М. С учетом данных о потерях, полученных в разрядах с повышенным полем и током, проанализирована регрессионная зависимость снижения уровня сигнала анализатора атомов перезарядки в канале с энергией, близкой к энергии инжекции нейтрального пучка, от относительной амплитуды возмущения, величины тороидального магнитного поля и тока по плазме. Обнаружена зависимость от амплитуды ТАЕ в степени порядка $\sim 0.5$ и обратная пропорциональность произведению магнитного поля и тока по плазме, которая, по всей видимости, определяется величиной тока по плазме, ввиду слабой зависимости от магнитного поля. Полученный результат свидетельствует о том, что потери быстрых ионов в токамаке Глобус-М2 вследствие возбуждения ТАЕ преимущественно обусловлены смещением траекторий пролетных частиц к границе плазмы. С повышением магнитного поля и тока также уменьшились провалы в потоке нейтронов в моменты вспышек тороидальных мод.
\end{abstract}

Ключевые слова: тороидальные альфвеновские моды, NPA, сферический токамак, потери быстрых ионов.

DOI: $10.21883 /$ JTF.2022.01.51850.227-21

\section{Введение}

Тороидальные альфвеновские моды (TAE) способны оказывать влияние на удержание быстрых частиц в токамаке, так как их возбуждение происходит за счет резонанса с быстрыми ионами, скорость которых порядка альфвеновской скорости $v_{A}=B_{0} / \sqrt{\mu_{0} \rho_{i}} \quad\left(B_{0}-\right.$ невозмущенное локальное магнитное поле, $\rho_{i}$ - массовая плотность ионов, $\mu_{0}-$ вакуумная магнитная проницаемость). Условие резонанса выполняется для частиц со скоростями $v_{A}$ и $v_{A} / 3[1,2]$. Источником энергии для возбуждения ТАЕ, как правило, являются ионы высокой энергии, образовавшиеся либо при перезарядке пучка нейтралей, инжектируемого в плазму (NBI), либо за счет ионно-циклотронного (ICRH) нагрева [2,3]. Сообщается также и о дестабилизации TAE в режимax с электрон-циклотронным (ECRH) нагревом [4]. TAE нередко наблюдались в плазме сферических токамаков: MAST [5], NSTX [6], START [7], Глобус-M [8,9], так как одним из параметров, влияющих на величину зазора, является аспектное отношение, и для токамаков с малым аспектным отношением величина частотного зазора, в котором могут существовать тороидальные моды, больше, чем для классических [10]. Альфвеновские моды оказывают существенное влияние на нейтронный выход не только из-за того, что могут приводить к окончательной потере быстрых частиц, но и в связи с тем, что приводят к их перераспределению как в пространстве скоростей (замедление), так и в физическом пространстве. Перенос быстрых частиц в периферийные области с более низкой температурой и концентрацией, во-первых, может уменьшать время замедления быстрых ионов, во-вторых, напрямую приводит к уменьшению нейтронного выхода из-за уменьшения числа актов реакций при низкой плотности. В классических токамаках именно ТАЕ представляют наибольшую опасность с точки зрения потерь быстрых ионов: потери, связанные с ними, могут достигать 70\% всех инжектированных в плазму ионов [3]. Для сферических токамаков потери быстрых ионов, связанные непосредственно с ТАЕ, менее исследованы. Так, в [11] сообщается о снижении потока нейтронов до $15 \%$ в NSTX вследствие так называемой альфвеновской лавины, а в [12] об уменьшении потока нейтронов на $25 \%$ во время fishbone-неустойчивости, развивающейся после ТАE в токамаке MAST. Потери, связанные непосредственно с отдельными ТАЕ в сферических токамаках, требуют отдельного рассмотрения.

Особенно важным оказывается изучение влияния ТАЕ на удержание быстрых ионов именно в контексте термоядерного источника нейтронов на основе сферического 
токамака, работу которого планируется осуществлять в квазинепрерывном режиме, т.е. с преимущественной долей тока, поддерживаемой неиндуктивными методами (нейтральной инжекцией, радиочастотной генерацией тока). Существенная часть нейтронного выхода такого источника должна быть обусловлена взаимодействием быстрых ионов, образующихся за счет инжекции нейтрального пучка, с тепловой плазмой и друг с другом. Не меньшее влияние на баланс энергии тороидальные моды могут оказывать в установках реакторного типа (ITER [3]), где за поддержание реакции отвечают термоядерные альфа-частицы, которые, достаточно замедлившись, также способны терять свою энергию в резонансе с альфвеновской волной. При этом вопрос предсказания величины потерь ионов, вызванных ТАЕ, кажется авторам недостаточно проработанным и требует отдельного исследования. Для анализа подобных зависимостей, как правило, используются два подхода, один из которых моделирование при помощи численных кодов (например, для анализа взаимодействия альфеновских мод с частицами используется код ASCOT [13]), а другой состоит в построении скейлингов или регрессий на основании большого числа экспериментальных данных. Каждый из этих подходов обладает своим набором достоинств и недостатков. Настоящая работа посвящена получению линейной регрессии для величины спада сигнала анализатора атомов перезарядки (NPA).

В разд. 1 описано устройство диагностического комплекса токамака Глобус-М2, применяемого в экспериментах по наблюдению ТАЕ. В разд. 2 получена регрессия для величины потерь быстрых ионов от амплитуды возмущения и параметров плазмы. Разд. 3 посвящен влиянию альфвеновских мод на нейтронный выход.

\section{1. Эксперимент на токамаке Глобус-М2}

Глобус-М2 - компактный (большой радиус $R=36 \mathrm{~cm}$, малый радиус $r=24 \mathrm{~cm}$, аспектное отношение $A=1.5$ ) сферический токамак $[14,15]$, который является модернизированной версией токамака Глобус-М $[15,16]$. Установка Глобус-М2 оборудована двумя $[17,18]$ инжекторами атомов высокой энергии мощностью до $1 \mathrm{MW}$ и энергией инжекции до 30 и $50 \mathrm{keV}$ соответственно. В состав диагностического комплекса токамака входят два анализатора атомов перезарядки (NPA): ACORD-12 и ACORD-24M [19]. Оба анализатора приспособлены для детектирования атомов как водорода, так и дейтерия, и имеют временное разрешение $0.1 \mathrm{~ms}$. ACORD-12 имеет 6 спектральных каналов для регистрации атомов водорода, настраиваемых в диапазоне $250-30 \mathrm{keV}$, и 6 каналов для регистрации атомов дейтерия в диапазоне $400-20 \mathrm{keV}$. Анализатор установлен в экваториальной плоскости нормально к центральному столбу. В свою очередь, ACORD-24M имеет по 12 каналов для регистрации атомов и водорода, и дейтерия, регулируемых в

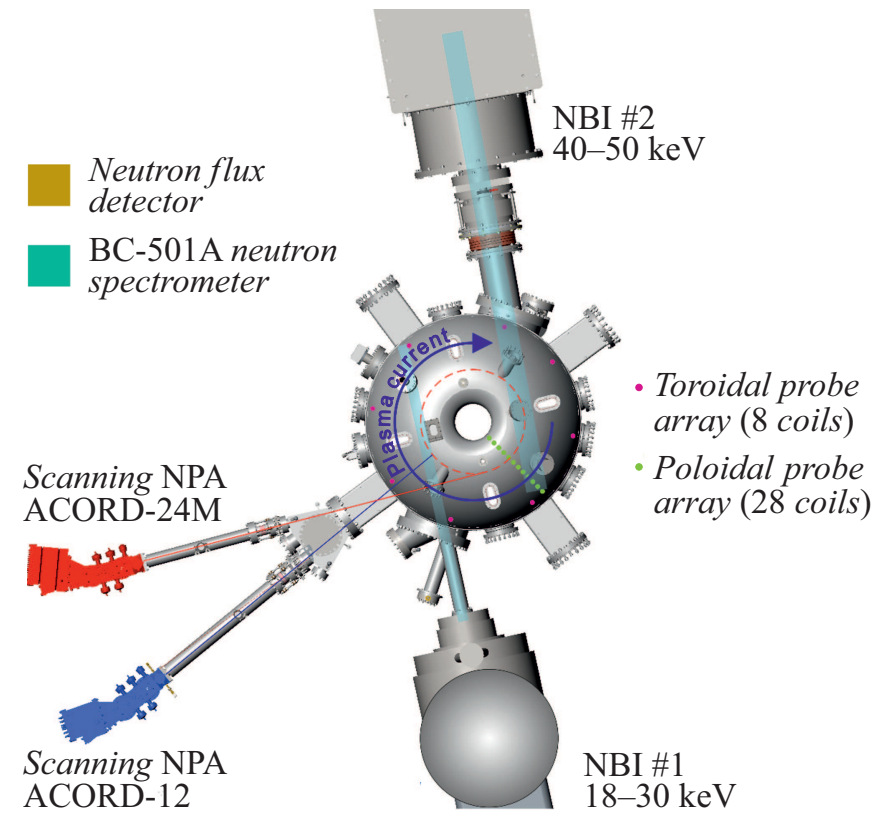

Рис. 1. Расположение инжекторов и диагностик, использовавшихся в эксперименте.

диапазоне $250-35 \mathrm{keV}$. Этот анализатор установлен тангенциально к окружности радиуса, равного прицельному параметру первого инжектора $(30 \mathrm{~cm})$. Поток нейтронов регистрируется при помощи ${ }_{5}^{10} B$ коронного счетчика, удаленного от камеры токамака на расстояние $12.7 \mathrm{~m}$, и нейтронного спектрометра на основе жидкого сцинтиллятора ВC-501A [20]. Для регистрации альфвеновских колебаний используется набор из 8 магнитных зондов, расположенных вдоль тороидального обхода на равных угловых расстояниях друг от друга, измеряющих радиальную компоненту магнитного потока. Схема расположения инжекторов и элементов диагностического комплекса установки Глобус-М2 показана на рис. 1.

В экспериментах на токамаке Глобус-М [9,21,22], где во время нейтральной инжекции наблюдались ТАЕ, использовался первый инжектор с энергией $E_{N B I}=28 \mathrm{keV}$ и мощностью $P_{\text {beam }}$ порядка $0.85 \mathrm{MW}$, а также анализатор ACORD-24M. Анализатор ACORD-12 использовался для измерений ионной температуры [23]. Отдельные вспышки ТАE, наблюдаемые на сигнале магнитных зондов, приводили к кратковременным просадкам в потоке нейтралей в канале, настроенном на регистрацию частиц с энергией, близкой к энергии инжекции $(28.5 \pm 1.5 \mathrm{keV})$. Измерения величины этих просадок производились с усреднением по окну $300 \mu \mathrm{s}$. Следует отметить, что провал в потоке нейтралей в канале анализатора не свидетельствует об окончательной потере этих частиц, а лишь об их перераспределении как в физическом пространстве, так и в пространстве скоростей. Однако, поскольку такие провалы наблюдаются также и на сигнале детектора нейтронов, такое перераспределение приводит к уменьшению нейтронного выхода, 
что особенно существенно для термоядерного источника нейтронов.

TAE в токамаке Глобус-М регистрировались в диапазоне параметров плазмы $\left\langle n_{e}\right\rangle<10^{20} \mathrm{~m}^{-3}$, $I_{p}=180-250 \mathrm{kA}, \quad B_{T}=0.4-0.5 \mathrm{~T} \quad$ как на стадии роста тока, так и на стадии плато [9]. В токамаке Глобус-М2 были достигнуты значения магнитного поля $B_{T}=0.6-0.8 \mathrm{~T}$ и тока по плазме до $400 \mathrm{kA}$, что позволило проанализировать зависимость потерь быстрых ионов в более широком диапазоне параметров, а ранее полученная $[21,22]$ зависимость была дополнена [24] соответствующими точками (рис. 5 в работе [21], рис. 1.1 в работе [24]). Точки, соответствующие более высоким значениям поля и тока, как и полагалось ранее, лежат на графике ниже точек, соответствующих более низким значениям, что свидетельствует об уменьшении потерь быстрых ионов. При этом для точек, соответствующих низким значениям поля $(0.4-0.5 \mathrm{~T})$, и тока $(0.18-0.25 \mathrm{kA})$ при больших значениях амплитуды возмущения (> 4G) характер зависимости меняется, и экспериментальные точки выходят на уровень насыщения, тогда как для более высоких значений поля $(0.6-0.8 \mathrm{~T})$ и тока (0.3-0.4 MА) такого насыщения не происходит. Точки, относящиеся к участку насыщения, были исключены из дальнейшего анализа.

\section{2. Анализ потерь быстрых ионов}

Искалась регрессионная зависимость относительной величины провала в потоке NPA от амплитуды TAE- $\delta B$, тороидального поля $B_{0}$ и тока по плазме $I_{p}$ в виде степенной функции $d N / N=C \delta B^{\alpha} B_{0}^{\beta} I_{p}^{\gamma}$, где $C-$ неизвестная постоянная. Величина провала в потоке нейтралей на анализатор $d N / N=\left(N_{1}-N_{2}\right) / N_{1}$, где $N_{1}$ и $N_{2}$ - число отсчетов анализатора до и после провала соответственно. Поскольку с повышением магнитного поля ток в большинстве разрядов повышался пропорционально с целью сохранения МГД-устойчивости разряда, эти величины не являются независимыми и обладают большим коэффициентом корреляции Пирсона $\rho_{\beta \gamma}=\operatorname{cov}\left(B_{0}, I_{p}\right) / \sigma\left(B_{0}\right) \sigma\left(I_{p}\right)=0.64$. В установке Глобус-М2 при полях $0.6-0.8 \mathrm{~T}$ и токах $0.3-0.4 \mathrm{MA}$ тороидальные моды стали развиваться в основном на стадии роста тока при трансформации моды из альфвеновских каскадов [25], возникающих на этой стадии разряда из-за обращения профиля $q(r)$ вследствие скинэффекта. Учет таких вспышек ТАЕ привел к уменьшению взаимной корреляции этих величин до $\rho_{\beta \gamma}=0.51$, так как на стадии роста тока при фиксированном поле TAE могут возникать в широком диапазоне токов по плазме. Однако это все равно не позволяет использовать эти величины независимо, поэтому для дальнейшего анализа использовалось произведение $B_{0} I_{p}$. Также для того, чтобы было возможно сопоставление амплитуд возмущений, возникающих в установках с различным
Коэффициенты корреляции Пирсона $\rho_{x y}$ для $\delta B$, магнитного поля и тока

\begin{tabular}{c|l|l|l}
\hline Параметры & $\delta B$ & $B$ & $I_{p}$ \\
\hline$\delta B$ & 1 & 0.25 & 0.36 \\
\hline$B$ & 0.25 & 1 & 0.52 \\
\hline$I_{p}$ & 0.36 & 0.52 & 1
\end{tabular}

магнитным полем (несмотря на то, что такое сопоставление и не проводилось), использовался безразмерный параметр $\delta B / B_{0}$. Коэффициенты корреляции Пирсона для трех параметров приведены в таблице. Результирующая регрессионная зависимость искалась в виде $d N / N=C\left(\delta B / B_{0}\right)^{\alpha}\left(B_{0} I_{p}\right)^{\beta}$.

Далее методом наименьших квадратов определялись степени $\alpha, \beta$, свободный параметр $C$ и дисперсии $\sigma_{\alpha}, \sigma_{\beta}$. Отдельные измерения величины провалов в потоке нейтралей считались неравноточными, распределенными согласно распределению Пуассона с дисперсией $\sigma_{i}$. Минимизировалась остаточная сумма квадратов $\chi^{2}$ :

$$
\chi^{2}=\sum_{i=1}^{N} g_{i}\left(\ln \left(\frac{d N}{N}\right)_{i}-\ln C-\alpha\left(\frac{\delta B}{B}\right)_{i}-\beta\left(B_{0} I_{p}\right)_{i}\right)^{2} .
$$

Для учета неравноточности измерений введены весовые коэффициенты $g_{i}=\sigma^{2} / \sigma_{i}^{2}$, где величина $\sigma^{2}=1 / \Sigma 1 / \sigma_{i}^{2}$ определяется из условия нормировки коэффициентов $g_{i}$ на единицу. Дисперсия отдельных измерений определялась как

$$
\sigma_{i}=\frac{\sqrt{\left(N_{1, i}-N_{2, i}\right)}}{N_{1, i}} .
$$

Дисперсии степеней $\sigma_{\alpha}$ и $\sigma_{\beta}$ определялись путем вычисления ковариационной матрицы для полученных оценок $\bar{\alpha}$ и $\bar{\beta}$ :

$$
\left(\begin{array}{cc}
\sigma_{\alpha}^{2} & \operatorname{cov}(\alpha, \beta) \\
\operatorname{cov}(\beta, \alpha) & \sigma_{\beta}^{2}
\end{array}\right)=2\left(\begin{array}{cc}
\frac{\partial^{2} \chi^{2}}{\partial \alpha^{2}} & \frac{\partial^{2} \chi^{2}}{\partial \alpha^{2} \beta} \\
\frac{\partial^{2} \chi^{2}}{\partial \beta \partial \alpha} & \frac{\partial^{2} \chi^{2}}{\partial \alpha^{2}}
\end{array}\right)_{\substack{\alpha=\bar{\alpha} \\
\beta=\bar{\beta}}} .
$$

Результирующая линейная регрессия для величины провалов в потоке анализатора $d N / N$ дается выражением

$$
\frac{d N}{N}=1.35 \cdot 10^{3}\left(\frac{\delta B}{B_{0}}\right)^{0.51 \pm 0.15}\left[B_{0} I_{p}\right]^{-0.94 \pm 0.27} .
$$

Указанные в показателях степеней ошибки соответствуют доверительному интервалу с заданным значением $3 \sigma$. Также полученная регрессия представлена на рис. 2. Следует отметить, что каждый из сомножителей в (4) входит как отдельный составной параметр, члены которого не являются независимыми переменными, что было показано в начале этого раздела. 


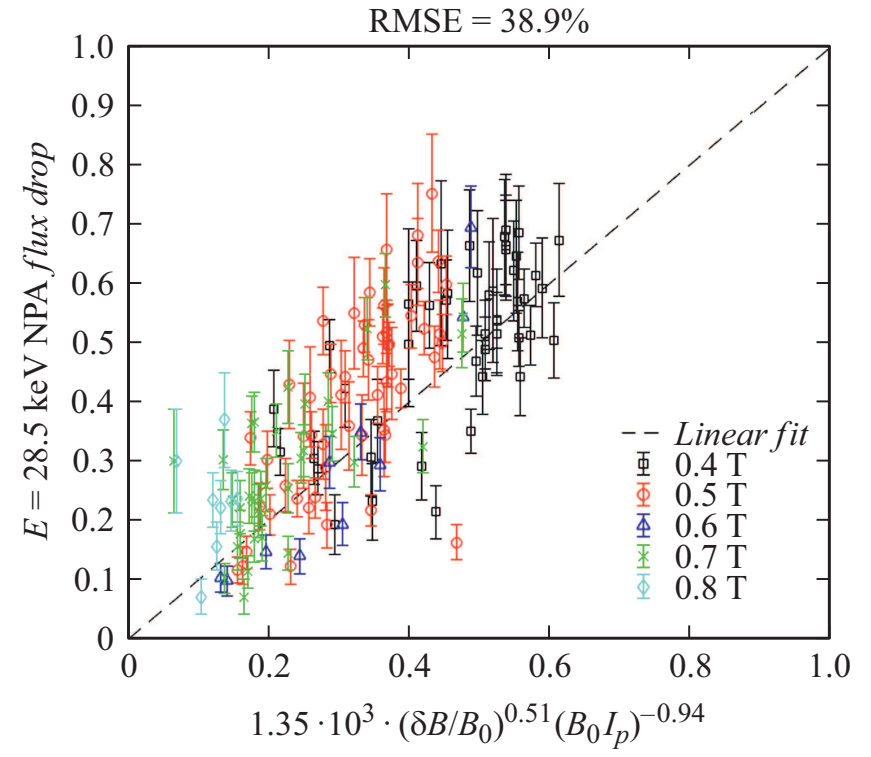

Рис. 2. Регрессионная зависимость величины провалов в потоке NPA от относительной амплитуды ТАЕ и произведения магнитного поля на ток по плазме.

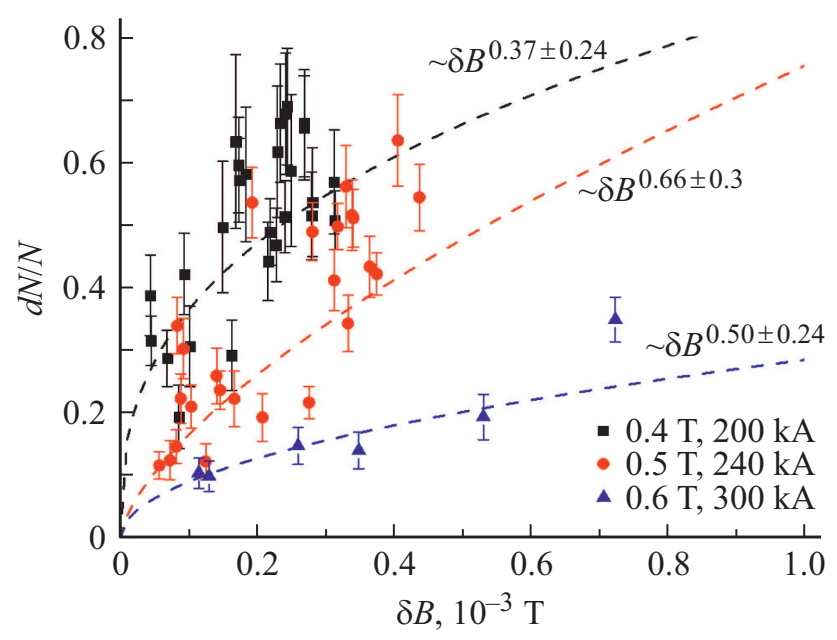

Pис. 3. Зависимость величины провалов в потоке NPA от амплитуды ТАЕ для разных значений магнитного поля и тока при одинаковом запасе устойчивости.

Однако, поскольку корреляция между величинами $\delta B$ и $B_{0}$ невысока (см. таблицу), аналогичным образом отдельно определялась зависимость величины $d N / N$ от непреведенной амплитуды $\delta B$ при фиксированных значениях магнитного поля и тока для трех групп точек из представленных на рис. 2: $0.4 \pm 0.02 \mathrm{~T}$ и $200 \pm 20 \mathrm{kA}, \quad 0.5 \pm 0.02 \mathrm{~T}$ и $240 \pm 20 \mathrm{kA}, \quad 0.6 \pm 0.02 \mathrm{~T}$ и $300 \pm 20 \mathrm{kA}$. Для больших значений поля и тока TAE с большими амплитудами по всей видимости не возбуждается, поэтому данные для полей $0.7-0.8 \mathrm{~T}$ и токов до $400 \mathrm{kA}$ не были использованы для анализа. Показатели степеней для зависимостей (рис. 3) величины провалов от $\delta B$ попадают в доверительный интервал определения показателя степени $\alpha$, в которой величина $\delta B / B_{0}$ входит в выражение (4). Из этого можно сделать вывод, что степень зависимости величины провалов $d N / N$ от амплитуды $\delta B$ порядка 0.5 , а зависимость от магнитного поля слабая. Тогда степень $\beta=-0.94$, в которой величина $B_{0} I_{p}$ (т.е. ток по плазме, если не учитывать слабую зависимость от магнитного поля) входит в выражение (4), свидетельствует о том, что потери быстрых ионов обусловлены преимущественно смещением траектории пролетных частиц вдоль большого радиуса. Смещение траектории пролетной частицы от магнитной поверхности (в дрейфовом приближении) можно оценить как $d=R-R_{c} \sim q \rho_{c i} \sim 1 / I_{p}$, где $R-$ положение центра орбиты пролетной частицы по большому радиусу, $R_{c}$ - радиус магнитной поверхности, где произошла ионизация, $\rho_{c i}-$ ионный ларморовский радиус, $q=q_{c y l}-$ коэффициент запаса устойчивости в цилиндрическом приближении. Таким образом, по полученной в (4) регрессии можно сделать вывод о том, что уменьшение потерь быстрых ионов обусловлено преимущественно увеличением тока по плазме, что подтверждает предположение, выдвинутое ранее в работе [9].

\section{3. Влияние альфвеновских мод на нейтронный выход}

В разрядах с альфвеновскими модами наблюдались не только просадки в сигнале анализатора атомов перезарядки, но и непосредственное уменьшение нейтронного выхода во время вспышек. На токамаке Глобус-М2 недавно были достигнуты значения магнитного поля до $0.85 \mathrm{~T}$ и тока по плазме до $400 \mathrm{kA}$. Это увеличение тока по плазме и магнитного поля также повлияло и на нейтронный выход. Ранее для токамака Глобус-М была получена [22] зависимость числа зарегистрированных нейтронов от амплитуды ТАЕ для поля $0.4 \mathrm{~T}$ и токов 180-230 kA по данным пропорционального счетчика нейтронов, заполненного ${ }_{2}^{3} \mathrm{He}$, ранее использовавшегося на установке Глобус-М. В связи с тем что диагностика нейтронов была усовершенствована (вместо пропорционального счетчика используется коронный счетчик и нейтронный спектрометр), сравнение ранее полученных данных с новыми оказывается затруднительно. Однако в экспериментах на установке Глобус-М2 при поле $0.5 \mathrm{~T}$ токе 200-250 kA также регистрировались тороидальные моды, возбуждение которых приводило к кратковременному снижению уровня сигнала нейтронного детектора. На рис. 4 представлены осциллограммы сигналов магнитных зондов (сверху) и зависимости числа отсчетов нейтронного спектрометра от времени во всем диапазоне энергий (снизу) для трех разрядов: \#39001 $(0.5 \mathrm{~T}, 209 \mathrm{kA}), \# 39027(0.6 \mathrm{~T}, 246 \mathrm{kA}), \# 38385(0.7 \mathrm{~T}$, $340 \mathrm{kA})$, в которых регистрировались вспышки ТАЕ примерно одинаковой амплитуды (порядка $10 \mathrm{G}$ ). Если для первых двух разрядов относительная величина про- 

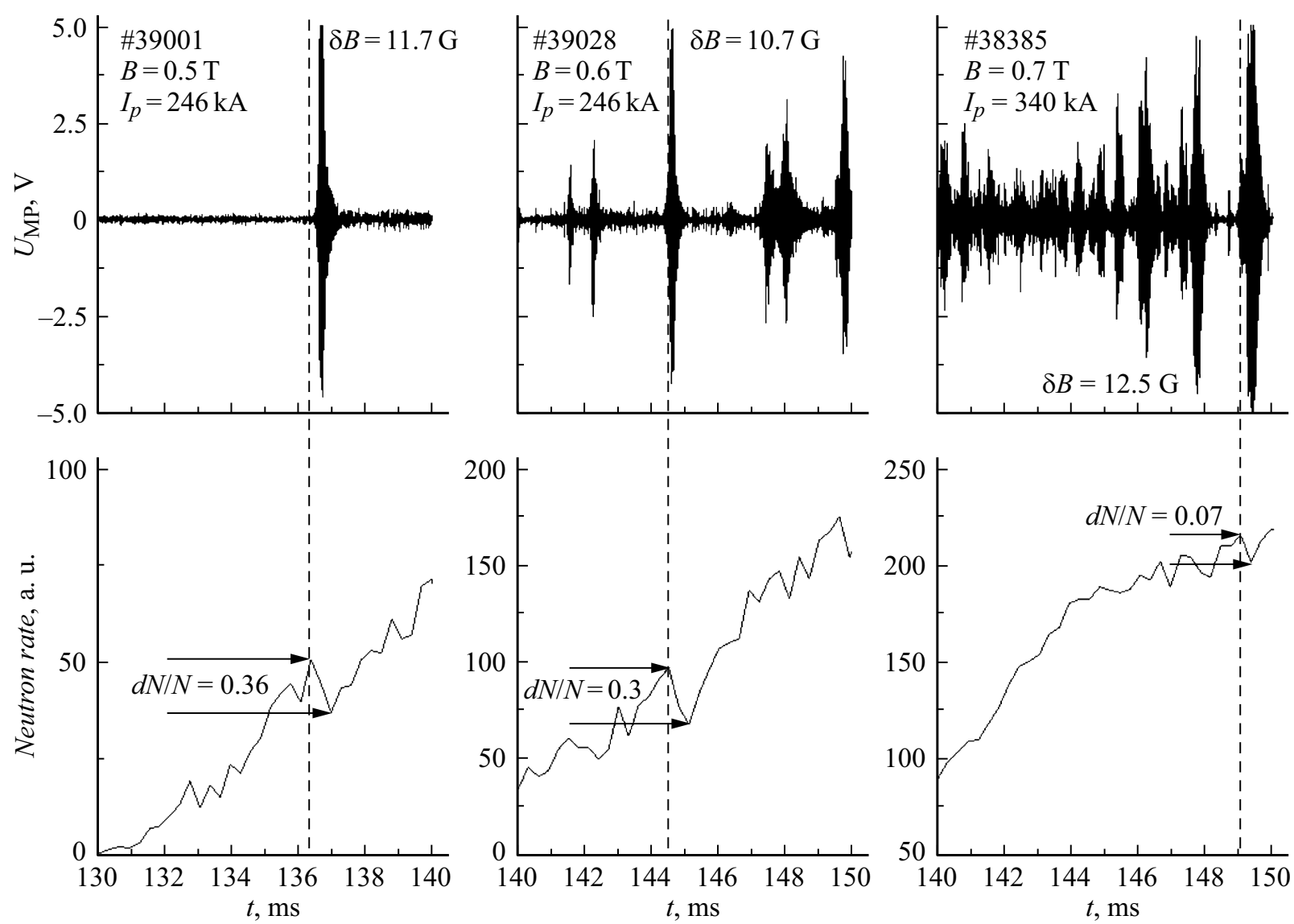

Рис. 4. Провалы в потоке нейтронов (снизу), вызываемые вспышками ТАЕ близкой амплитуды для разрядов с разными значениями магнитного поля и тока. Слева направо: разряд \#39001 (0.5 T, $209 \mathrm{kA})$, разряд \#39028 (0.6 T, $246 \mathrm{kA})$ и разряд \#38385 (0.7 Т, $340 \mathrm{kA})$.

вала составляет около 30\%, то для разряда \#38385 эта величина порядка 7\%. Такие провалы оказываются слабо различимы на фоне флуктуаций сигнала нейтронного детектора, что накладывает ограничения на использование нейтронной диагностики для анализа потерь быстрых ионов, связанных с распространением ТАЕ.

\section{4. Результаты и заключение}

В настоящей работе был проанализирован характер зависимости потерь быстрых ионов от амплитуды тороидальных альфвеновских мод и параметров плазмы. Ввиду сильной корреляции магнитного поля и тока получена регрессионная зависимость от относительной амплитуды ТАЕ $\left(\delta B / B_{0}\right)$ и произведения $B_{0} I_{p}$. Показано, что степень зависимости от величины $\delta B / B_{0} \sim 0.5$ и порядка $\sim-1$ от величины $B_{0} I_{p}$, а зависимость от $B_{0}$ более слабая, чем от $\delta B$ и от тока. При этом разделить зависимость от магнитного поля и от тока затруднительно, так как большинство разрядов установки Глобус-М при низких значениях поля и тока производились с сохранением запаса устойчивости, что привело к высокому значению корреляции этих величин. Однако полностью пренебречь зависимостью от магнитного поля также не получается, поскольку приведенная оценка для смещения орбиты получена в приближении малого аспектного отношения и, разумеется, в полной мере не отражает изменение траекторий орбит в сферическом токамаке. Тем не менее полученный результат указывает на то, что основной причиной снижения потерь в установке Глобус-М2 по сравнению с установкой Глобус-М может являться уменьшение величины смещения орбит пролетных частиц от магнитных поверхностей вдоль большого радиуса с ростом тока по плазме, как это и происходит в классических токамаках. Поэтому возмущения с такой же амплитудой более не приводят к потерям частиц изза перехода на орбиты, где они не удерживаются.

Для того чтобы разделить влияние тока по плазме и тороидального магнитного поля на потери быстрых ионов, на токамаке Глобус-М2 запланирована серия экспериментов по наблюдению ТАЕ со сканированием в широком диапазоне магнитных полей и токов.

Дальнейший анализ потерь методом, описанным в настоящей работе, при более высоких значениях поля и тока (планируется увеличения магнитного поля до $1 \mathrm{~T}$ и тока до 0.5 МА) может оказаться затруднительным, так как, во-первых, уже при значениях 0.7 T, 300 kA TAE не приводят к сколь-либо заметным потерям (наиболее хорошо это видно на сигнале нейтронного спектро- 
метра - рис. 4), а во-вторых, наблюдается изменение характера развития самих ТАЕ. В токамаке Глобус-М2 TAE развиваются в соответствии с моделью хищникжертва [9]: большие коэффициенты нарастания вызывают стремительный рост амплитуды колебания, что вызывает перераспределение частиц, и, в свою очередь, быстрое затухание. При таком сценарии относительно легко идентифицировать просадки сигнала анализатора атомов перезарядки. При больших значениях тока по плазме смещение траекторий пролетных частиц к центру плазменного шнура больше, и возбуждение ТАЕ не приводит к изменению характера траекторий. Вследствие этого время жизни отдельных реализаций ТАЕ увеличивается, их амплитуда уменьшается, и отчетливых просадок как на сигнале нейтрального анализатора, так и в потоке нейтронов не наблюдается. Достаточно большое значение среднеквадратического отклонения (38\%) может говорить о том, что присутствуют скрытые зависимости от иных параметров плазмы, а также от предшествующих событий, приводивших к перераспределению частиц в фазовом пространстве.

\section{Финансирование работы}

Исследование потерь быстрых ионов в разд. 2 выполнено при поддержке Российского научного фонда (проект № 17-12-01177-П). Измерение потока нейтронов, описанные в разд. 3 выполнено при поддержке Министерства науки и высшего образования Российской Федерации в рамках государственного задания по проекту № 0034-2021-0001. Измерения основных параметров плазмы были проведены на уникальной научной установке „Сферический токамак Глобус-М“, входящей в состав Федерального центра коллективного пользования „Материаловедение и диагностика в передовых технологиях“ (проект RFMEFI62119X0021) в рамках государственного задания по проекту № 0040-2019-0023 в ФТИ им. А.Ф. Иоффе.

\section{Конфликт интересов}

Авторы заявляют, что у них нет конфликта интересов.

\section{Список литературы}

[1] W.W. Heidbrink. Phys. Plasmas, 15, 055501 (2008). DOI: $10.1063 / 1.2838239$

[2] K.-L. Wong. Plasma Phys. Control. Fusion, 41, R1 (1999). DOI: 10.1088/0741-3335/41/1/001

[3] ITER Physics Expert Group on Energetic Particles, Heating and Current Drive and ITER Physics Basis Editors, Nucl. Fusion, 39, 2471 (1999). DOI: 10.1088/0029-5515/39/12/305

[4] S.E. Sharapov, M. Garcia-Munoz, M.A. Van Zeeland, B. Bobkov, I.G.J. Classen, J. Ferreira, A. Figueiredo, M. Fitzgerald, J. Galdon-Quiroga, D. Gallart, B. Geiger, J. Gonzalez-Martin, T. Johnson, P. Lauber, M. Mantsinen, F. Nabais, V. Nikolaeva, M. Rodriguez-Ramos, L. SanchisSanchez, P.A. Schneider, A. Snicker, P. Vallejos and the AUG
Team and the EUROfusion MST1 Team11. Plasma Phys. Control. Fusion, 60, 014026 (2018). DOI: 10.1088/13616587/aa90ee

[5] M.P. Gryaznevich, S.E. Sharapov. Plasma Phys. Controlled. Fusion, 46, S15 (2004). DOI: 10.1088/0741-3335/46/7/S02

[6] E.D. Fredrickson, C.Z. Cheng, D. Darrow, G. Fu, N.N. Gorelenkov, G. Kramer, S.S. Medley, J. Menard, L. Roquemore, D. Stutman, R.B. White. Phys. Plasmas, 16, 2852 (2003). DOI: 10.1063/1.1579493

[7] M.P. Gryaznevich, S.E. Sharapov. Nucl. Fusion, 40, 907 (2000). DOI: $10.1088 / 0029-5515 / 40 / 5 / 303$

[8] В.В. Буланин, В.К. Гусев, Г.С. Курскиев, В.Б. Минаев, М.И. Патров, А.В. Петров, М.А. Петров, Ю.В. Петров, А.Ю. Тельнова, А.Ю. Яшин. Письма в ЖТФ, 43 (23), 40 (2017). DOI: 10.21883/PJTF.2017.23.45274.16986 [V.V. Bulanin, V.K. Gusev, G.S. Kurskiev, V.B. Minaev, M.I. Patrov, A.V. Petrov, M.A. Petrov, Yu.V. Petrov, A.Yu. Tel'nova, A.Yu. Yashin. Tech. Phys. Lett., 43, 1067 (2017) DOI: 10.1134/S1063785017120033]

[9] Ю.В. Петров, Н.Н. Бахарев, В.В. Буланин, В.К. Гусев, Г.С. Курскиев, А.А. Мартынов, С.Ю. Медведев, В.Б. Минаев, М.И. Патров, А.В. Петров, Н.В. Сахаров, П.Б. Щеголев, А.Ю. Тельнова, С.Ю. Толстяков, А.Ю. Яшин. Физика плазмы, $45(8), 675$ (2019). DOI: 10.1134/S0367292119080080 [Yu.V. Petrov, N.N. Bakharev, V.V. Bulanin, V.K. Gusev, G.S. Kurskiev, A.A. Martynovc, S.Yu. Medvedev, V.B. Minaev, M.I. Patrov, A.V. Petrov, N.V. Sakharov, P.B. Shchegolev, A.Yu. Telnova, S.Yu. Tolstyakov, A.Yu. Yashin. Plasma Phys. Rep., 25, 723 (2019) DOI: 10.1134/S1063780X19080075]

[10] N.F. Cramer. The Physics of Alfven Waves (WILEY-VCH Verlag, Berlin, 2001), p. 201-204.

[11] E.D. Fredrickson, N.A. Crocker, D.S. Darrow, N.N. Gorelenkov, G.J. Kramer, S. Kubota, M. Podesta, R.B. White, A. Bortolon, S.P. Gerhardt, R.E. Bell, A. Diallo, B. LeBlanc, F.M. Levinton, H. Yuh. Nucl. Fusion, 53, 013006 (2013). DOI: 10.1088/0029-5515/53/1/013006

[12] O.M. Jones, M. Cecconello, K.G. McClements, I. Klimek, R.J. Akers, W.U. Boeglin, D.L. Keeling, A.J. Meakins, R.V. Perez, S.E. Sharapov, M. Turnyanskiy and the MAST team. Plasma Phys. Control. Fusion, 57, 125009 (2015). DOI: 10.1088/0741-3335/57/12/125009

[13] E. Hirvijoki, A. Snicker, T. Korpilo, P. Lauber, E. Poli, M. Schneller, T. Kurki-Suonio. Comp. Phys. Communications, 183, 2589 (2012). DOI: 10.1016/j.cpc.2012.07.009

[14] V.B. Minaev, V.K. Gusev, N.V. Sakharov, V.I. Varfolomeev, N.N. Bakharev, V.A. Belyakov, E.N. Bondarchuk, P.N. Brunkov, F.V. Chernyshev, V.I. Davydenko, V.V. Dyachenko, A.A. Kavin, S.A. Khitrov, N.A. Khromov, E.O. Kiselev. Nucl. Fusion, 57, 066047 (2017). DOI: $10.1088 / 1741-4326 / \mathrm{aa} 69 \mathrm{e} 0$

[15] V.K. Gusev, N.N. Bakharev, V.A. Belyakov, B.Ya. Ber, E.N. Bondarchuk, V.V. Bulanin, A.S. Bykov, F.V. Chernyshev, E.V. Demina, V.V. Dyachenko, P.R. Goncharov, A.E. Gorodetsky, E.Z. Gusakov, A.D. Iblyaminova, A.A. Ivanov. Nucl. Fusion, 55, 104016 (2015). DOI: 10.1088/0029-5515/55/10/104016

[16] В.К. Гусев, В.Е. Голант, Е.3. Гусаков, В.В. Дьяченко, М.А. Ирзак, В.Б. Минаев, Е.Е. Мухин, А.Н. Новохацкий, К.А. Подушникова, Г.Т. Раздобарин, Н.В. Сахаров, Е.Н. Трегубова, В.С. Узлов, О.Н. Щербинин, 
В.А. Беляков, А.А. Кавин, Ю.А. Косцов, Е.Г. Кузьмин, В.Ф. Сойкин, Е.А. Кузнецов, В.А. Ягнов. ЖТФ, 69 (9), 58 (1999). [V.K. Gusev, V.E. Golant, E.Z. Gusakov, V.V. D’yachenko, M.A. Irzak, V.B. Minaev, E.E. Mukhin, A.N. Novokhatskii, K.A. Podushnikova, G.T. Razdobarin, N.V. Sakharov, E.N. Tregubova, V.S. Uzlov, O.N. Shcherbinin, V.A. Belyakov, A.A. Kavin, Yu.A. Kostsov, E.G. Kuz'min, V.F. Soikin, E.A. Kuznetsov, V.A. Yagnov. Tech. Phys., 44, 1054 (1999). DOI: 10.1134/1.1259469]

[17] В.К. Гусев, А.В. Деч, Л.А. Есипов, В.Б. Минаев, А.Г. Барсуков, Г.Б. Игонькина, В.В. Кузнецов, А.А. Панасенков, М.М. Соколов, Г.Н. Тилинин, А.В. Лупин, В.К. Марков. ЖТФ, 77 (9), 28 (2007). [V.K. Gusev, A.V. Dech, L.A. Esipov, V.B. Minaev, A.G. Barsukov, G.B. Igon'kina, V.V. Kuznetsov, A.A. Panasenkov, M.M. Sokolov, G.N. Tilinin, A.V. Lupin, V.K. Markov. Tech. Phys., 52, 1127 (2007) DOI: $10.1134 / \mathrm{S} 1063784207090058]$

[18] A.Yu. Telnova, V.B. Minaev, P.B. Shchegolev, N.N. Bakharev, I.V. Shikhovtsev, V.I. Varfolomeev. J. Phys.: Conf. Ser., 1400, 077015 (2019). DOI: 10.1088/1742-6596/1400/7/077015

[19] N.N. Bakharev, F.V. Chernyshev, P.R. Goncharov, V.K. Gusev, A.D. Iblyaminova, V.A. Kornev, G.S. Kurskiev, A.D. Melnik, V.B. Minaev, M.I. Mironov, M.I. Patrov, Yu.V. Petrov, N.V. Sakharov, P.B. Shchegolev, S.Yu. Tolstyakov. Nucl. Fusion, 55, 043023 (2015). DOI: 10.1088/0029-5515/55/4/043023

[20] M.V. Iliasova, A.E. Shevelev, E.M. Khilkevitch, I.N. Chugunov, V.B. Minaev, D.B. Gin, D.N. Doinikov, I.A. Polunovsky, V.O. Naidenov, M.A. Kozlovskiy, M.F. Kudoyarov. Nucl. Instr. and Methods in Phys. Research, Section A, 983, 164590 (2020). DOI: 10.1016/j.nima.2020.164590

[21] Н.Н. Бахарев, И.М. Балаченков, В.И. Варфоломеев, А.В. Воронин, В.К. Гусев, В.В. Дьяченко, М.В. Ильясова, Е.О. Киселев, А.Н. Коновалов, Г.С. Курскиев, А.Д. Мельник, В.Б. Минаев, И.В. Мирошников, А.Н. Новохацкий, М.И. Патров, Ю.В. Петров, Н.В. Сахаров, О.М. Скрекель, А.Ю. Тельнова, В.А. Токарев, С.Ю. Толстяков, Е.А. Тюхменева, Е.М. Хилькевич, Н.А. Хромов, Ф.В. Чернышев, И.Н. Чугунов, А.Е. Шевелев, П.Б. Щеголев. Физика плазмы, 46 (7), 579 (2020). DOI: $10.31857 / \mathrm{S} 036729212007001 \mathrm{X}$ [N.N. Bakharev, I.M. Balachenkov, F.V. Chernyshev, I.N. Chugunov, V.V. Dyachenko, V.K. Gusev, M.V. Iliasova, E.M. Khilkevitch, N.A. Khromov, E.O. Kiselev, A.N. Konovalov, G.S. Kurskiev, V.B. Minaev, A.D. Melnik, I.V. Miroshnikov, A.N. Novokhatsky, M.I. Patrov, Yu.V. Petrov, N.V. Sakharov, P.B. Shchegolev, A.E. Shevelev, O.M. Skrekel, A.Yu. Telnova, V.A. Tokarev, S.Yu. Tolstyakov, E.A. Tukhmeneva, V.I. Varfolomeev, A.V. Voronin. Plasma Phys. Rep., 46, 675 (2020). DOI: 10.1134/S1063780X20070016]

[22] Yu.V. Petrov, N.N. Bakharev, V.K. Gusev, V.B. Minaev, V.A. Kornev, G.S. Kurskiev, M.I. Patrov, N.V. Sakharov, S.Yu. Tolstyakov, P.B. Shchegolev. J. Plasma Phys., 81, 515810601 (2015). DOI: 10.1017/S0022377815001129

[23] N.N. Bakharev, F.V. Chernyshev, V.K. Gusev, E.O. Kiselev, G.S. Kurskiev, M.M. Larionova, A.D. Melnik, V.B. Minaev, M.I. Mironov, I.V. Miroshnikov, Yu.V. Petrov, N.V. Sakharov, P.B. Shchegolev, O.M. Skrekel, A.Yu. Telnova, E.A. Tukhmeneva, V.I. Varfolomeev. Plasma Phys. Control. Fusion, 62, 125010 (2020). DOI: 10.1088/1361-6587/abbe32
[24] I.M. Balachenkov, Yu.V. Petrov, V.K. Gusev, N.N. Bakharev, V.V. Bulanin, V.I. Varfolomeev, V.V. Dyachenko, N.S. Zhiltsov, E.O. Kiselev, A.N. Konovalov, S.V. Krikunov, G.S. Kurskiev, V.B. Minaev, M.I. Patrov, A.V. Petrov, A.M. Ponomarenko, N.V. Sakharov, A.Yu. Telnova, P.B. Shchegolev, A.Yu. Yashin. J. Phys.: Conf. Ser. 1697, 012212 (2020). DOI: 10.1088/17426596/1697/1/012212

[25] И.М. Балаченков, Ю.В. Петров, В.К. Гусев, Н.Н. Бахарев, В.В. Буланин, В.И. Варфоломеев, Н.С. Жильцов, Е.О. Киселев, Г.С. Курскиев, В.Б. Минаев, М.И. Патров, А.В. Петров, А.М. Пономаренко, Н.В. Сахаров, А.Ю. Тельнова, В.А. Токарев, Н.А. Хромов, П.Б. Щеголев, А.Ю. Яшин. Письма в ЖТФ, 46 (23), 3 (2020). DOI: 10.21883/PJTF.2020.23.50338.18450 [I.M. Balachenkov, Yu.V. Petrov, V.K. Gusev, N.N. Bakharev, V.V. Bulanin, V.I. Varfolomeev, N.S. Zhil'tsov, E.O. Kiselev, G.S. Kurskiev, V.B. Minaev, M.I. Patrov, A.V. Petrov, A.M. Ponomarenko, N.V. Sakharov, A.Yu. Tel'nova, V.A. Tokarev, N.A. Khromov, P.B. Shchegolev, A.Yu. Yashin. Tech. Phys. Lett., 46, 1157 (2020). DOI: 10.1134/S1063785020120032 